\title{
The effect of simvastatin on warfarin anticoagulation: a Swedish register-based nationwide cohort study
}

\author{
Marine L. Andersson ${ }^{1} \cdot$ Buster Mannheimer ${ }^{2,3}$ (D) Jonatan D. Lindh ${ }^{1}$
}

Received: 13 March 2019 / Accepted: 30 May 2019 / Published online: 14 June 2019

(C) The Author(s) 2019

\begin{abstract}
Purpose Some data indicate that simvastatin may increase the anticoagulative effect in patients treated with warfarin, but the evidence is scarce. The aim of the present study was to investigate how the anticoagulative effect of warfarin is affected by the initiation of simvastatin in a very large patient sample.

Methods In a retrospective cohort study, we included 5637 individuals on warfarin treatment initiating simvastatin. INR values and warfarin doses were calculated week-by-week during co-treatment. Data were obtained from two large Swedish warfarin registers and from the Swedish Prescribed Drug Register.

Results INR increased from 2.43 at baseline to $2.58,4$ weeks after simvastatin initiation, and did not stabilize until the last quarter of the year studied. Consequently, the proportion of patients with an INR above 3 increased from around 8 to $15 \%$.

Conclusions In conclusion, initiation of simvastatin resulted in moderately increased INR values and subsequent dose decreases in patients already on warfarin. In order to avoid the increased risk of bleeding, the initiation of simvastatin may be accompanied by closer INR monitoring.
\end{abstract}

Keywords Simvastatin $\cdot$ Cytochrome P-450 CYP2C9 $\cdot$ Drug interactions $\cdot$ International normalized ratio $\cdot$ Warfarin

\section{Introduction}

Warfarin is the world-leading oral anticoagulant used for the treatment and prevention of thromboembolic disease. The therapeutic interval of warfarin is narrow, and the dose needed for sufficient anticoagulation is close to that which may cause bleeding [1]. The effect of warfarin is influenced by genetic factors, for example, vitamin $\mathrm{K}$ epoxide reductase complex (VKORC1) and cytochrome P450 2C9 (CYP2C9) genotypes,

Buster Mannheimer

buster.mannheimer@sll.se

1 Karolinska Institutet, Department of Laboratory Medicine, Division of Clinical Pharmacology, Karolinska University Hospital Huddinge, Stockholm, Sweden

2 Department of Clinical Science and Education at Södersjukhuset, Karolinska Institutet, Stockholm, Sweden

3 Section of Diabetes and Endocrinology, Department of Internal Medicine, Södersjukhuset, Sjukhusbacken 8-10, 118 82 Stockholm, Sweden but also exogenic factors such as dietary vitamin $\mathrm{K}$ intake and drug interactions [2]. There are many examples of clinically relevant drug-drug interactions, such as increased warfarin effect due to amiodarone co-treatment $[3,4]$ and induction of CYP2C9 by carbamazepine [5] leading to subtherapeutic international normalized ratio (INR) levels.

Simvastatin is one of the most prescribed drugs and is often used by individuals also dispensed anticoagulants. In Sweden alone, about 50,000 individuals (428 per 100,000 inhabitants) are treated with simvastatin in combination with warfarin [6]. Some data indicate that simvastatin may increase the anticoagulative effect in patients treated with warfarin [3, 7-9]. We have previously studied the influence of the CYP2C9 genotype on the magnitude of the interaction between warfarin and simvastatin. In carriers of the CYP2C $9 * 3$ allele, simvastatin reduced warfarin dose requirements by $29 \%$, compared with $5 \%$ in non-carriers, suggesting that the mechanism may be due to selective inhibition of the inhibition of the CYP2C9*3 allele [10]. The main aim of the present study was to investigate how the anticoagulative effect of warfarin is affected by the initiation of simvastatin in a very large patient sample. 


\section{Methods}

In this retrospective cohort study, we hypothesized firstly that simvastatin would enhance the anticoagulative effect of warfarin and, secondly, that the effect would be more pronounced in patients with low maintenance doses, a group where CYP2C $9 * 3$ carriers are more common.

We investigated the change in warfarin dose and INR during simvastatin therapy in patients with ongoing warfarin anticoagulation, by comparing warfarin doses before and after initiation of co-treatment with simvastatin. Data on daily warfarin dose and results from INR measurements were retrieved from two anticoagulation registers, Journalia and Auricula $[11,12]$. These systems are used in more than 300 anticoagulation clinics in Sweden and contain information about warfarin doses, INR, sex, and personal identification numbers for patients using warfarin for atrial fibrillation as well as for other indications. Information on the use of other medications was retrieved from the Swedish Prescribed Drug Register [13]. This register includes data on all dispensed prescriptions in Sweden. By linking and matching these registers, we could analyze warfarin doses and INR in patients initiating simvastatin therapy.

All adult patients (age 18 or above) dispensed warfarin and starting simvastatin therapy were available for inclusion. The index date for the start of simvastatin therapy was defined as the date of the first simvastatin dispensing. To make sure simvastatin therapy was initiated, this period should have been preceded by a period of at least 12 months during which no simvastatin had been dispensed. Ongoing warfarin anticoagulation was defined by at least one dispensing of warfarin within 4 to 20 weeks before the index date and warfarin doses documented in Journalia or Auricula during the baseline period (420 weeks prior to the index date). To avoid including patients ending simvastatin therapy within a year, we also required three additional simvastatin dispensings after the index date, the first within 55-145 days after the index date, the second within 155-245 days, and the third within 255-345 days after the index date. In Sweden, each dispensing of simvastatin usually covers 3 months.

Patients were excluded if they were using other drugs known to influence warfarin pharmacokinetics, causing a more than $10 \%$ change in warfarin doses. Hence, patients were excluded if they had been dispensed any of the following drugs: amiodarone, bosentan, capecitabine, carbamazepine, cimetidine, clofibrate, co-trimoxazole, dabrafenib, darunavir, dasabuvir, disulfiram, dronedarone, enzalutamide, eslicarbazepine, erythromycin, fluconazole, fluorouracil, lopinavir, metronidazole, miconazole, paritaprevir, phenobarbital, primidone, propafenone, rifampicin, ritonavir, sitaxentan, ombitasvir, oritavancin, vemurafenib, voriconazole, or zafirlukast.
In the main analysis, we compared the mean daily warfarin dose 1-28 days prior to the index date (initiation of simvastatin) with the mean daily warfarin dose 337-364 days after the index date. In subjects ending warfarin therapy during the follow-up period, all available doses within the period were included in the analysis. The two periods were compared by calculation of the change in log-transformed dose in each patient, and the mean difference was compared with no change (zero) using a two-sided paired $t$ test. The relative dose decrease was calculated by retransformation of the mean difference.

A multiple regression model was used to investigate the effect of sex and age on the proposed drug-drug interaction. The dependent variable in the model was the change in log-transformed warfarin dose, and age was analyzed in groups that ranged 18-49, 50-59, 60$69,70-79,80-89$, and $90-100$ years. The dose at baseline was plotted against the dose at week 49-52 in each patient to visualize the changes within the whole study population.

To study the interaction effect over time, we calculated the week-by-week normalized dose (by dividing dose by baseline dose) for each patient. Normalization was performed since we assumed that the relative effect (the percent dose change) would be more uniform than the change in dose/week in milligrams. We also calculated the fraction of patients with a decrease in warfarin dose by $>$ $10 \%,>25 \%$, and $>50 \%$ compared with baseline. The fraction was calculated separately for each week and not cumulatively. To investigate the theoretical possibility of time-dependent covariates reducing the correlation with baseline values over time, we also analyzed the proportion of patients with different levels of increases of warfarin doses after the index date. For symmetry reasons, a $10 \%$ decrease was contrasted against an $11 \%$ decrease, a $25 \%$ decrease against a $33 \%$ increase, and a $50 \%$ decrease against a $100 \%$ increase.

The effect on INR was investigated by interpolation of INR values by the method of Rosendaal [14]. All values were logtransformed and the mean INR value with $95 \%$ CI was calculated week by week until 52 weeks after the index date. We also calculated the fraction of patients having an INR above 3 and above 4 .

To investigate the effect of initial warfarin dose requirements, as a rough marker for the CYP2C $9 * 3$ genotype, we divided the patients into 4 subgroups based on the baseline dose (quartiles). All analyses described above were then repeated separately for each of these subgroups. In addition, we performed subgroup analyses based on the simvastatin dose. In this case, we divided patients into those receiving 10 $20 \mathrm{mg}$ simvastatin and those receiving $40-80 \mathrm{mg}$.

$p$ values below 0.05 were considered statistically significant. All analyses were performed using R version 3.3.2 [15]. 


\section{Results}

We identified 7305 patients during the period July 1, 2005, until December 31, 2012, who had started simvastatin therapy and who had been dispensed simvastatin three times after the index date according to the inclusion criteria and who had not received any interacting drugs. After further exclusions due to missing warfarin dosage data or lack of warfarin exposure 90 days or more before starting simvastatin, 5637 patients were included in the analysis (Fig. 1).

The median age of the included patients was 72 years (interquartile range (IQR) 65-78 years), and 34\% were female. The median warfarin dose per week during the baseline period was $32.5 \mathrm{mg}$ (IQR 23.8-42.5).

INR increased from 2.43 at baseline to $2.58,4$ weeks after simvastatin initiation, and did not stabilize until the last quarter of the year studied. Even then, the mean INR level had not returned to its baseline value slightly above 2.4 (Fig. 2). Figure 3 shows the fraction of patients with INR above 3 and above 4 . Fifteen percent of patients reached an INR above 3 and less than $1 \%$ an INR above 4 .

When comparing warfarin doses before simvastatin initiation with doses during a 4-week period (337-364 days) after simvastatin initiation, we found a decrease in warfarin doses by $6.8 \%$. In a multivariable regression model, we did not find

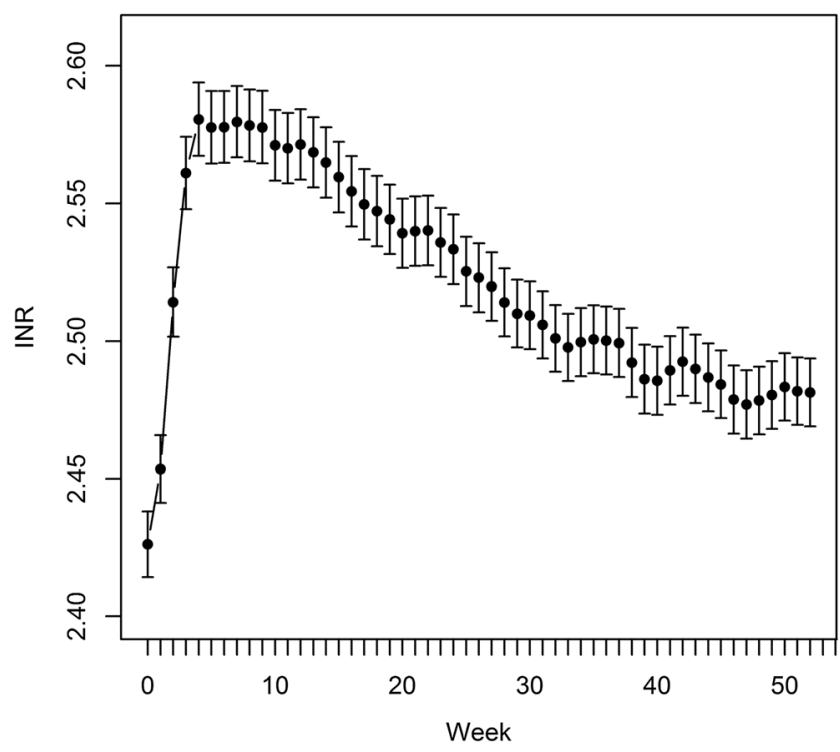

Fig. 2 Weekly mean INR during co-treatment with warfarin and simvastatin. The INR was interpolated to allow inclusion of weekly values for all patients. Brackets denote $95 \%$ confidence intervals

a significant effect of age or gender on the decrease in warfarin dose. The relative warfarin doses decreased slowly during the whole year studied (Fig. 4). The fraction of patients with a $>$ 10 decrease, $>25 \%$ decrease, and $>50 \%$ decrease in warfarin dose is presented in Fig. 5. Up to 35\% of patients experienced

Fig. 1 Patient flow diagram

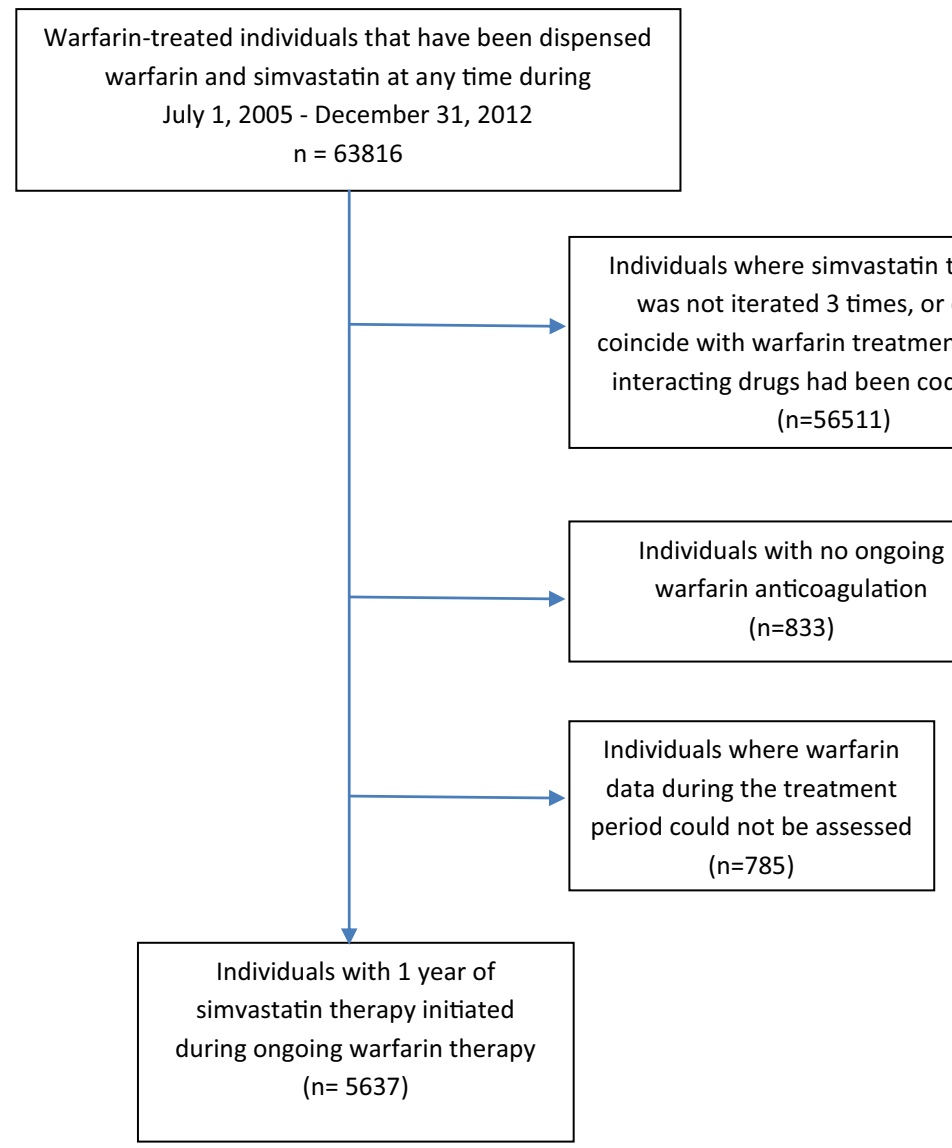




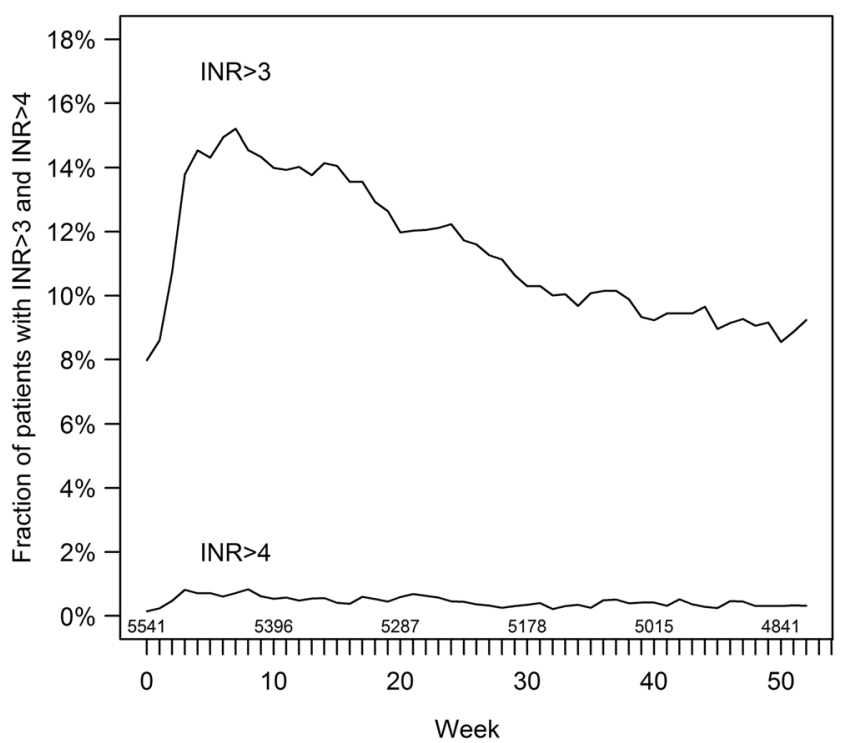

Fig. 3 Fraction of patients with an INR over 3.0 and 4.0 following simvastatin initiation. The numbers at the bottom of the graph indicate the number of patients remaining in the study (i.e., still co-treated with warfarin and simvastatin) at different time points

dose decreases by at least $10 \%$, and in approximately $5 \%$, the dose was decreased by $25 \%$ or more.

In addition, we performed subgroup analysis in patients with different simvastatin doses and baseline doses of warfarin. Simvastatin in doses $20 \mathrm{mg}$ and above was associated with a significant attenuated decreased warfarin dose $(-6.1 \%$, $p<0.01)$. The decreased warfarin dose associated with simvastatin doses of $40 \mathrm{mg}$ or above was more attenuated ($8.1 \%, p<0.001)$.

Analysis of the effect in individuals with different baseline doses of warfarin revealed a mean decrease by $5.6 \%$ in the subjects whose baseline dose was within the first quantile, by

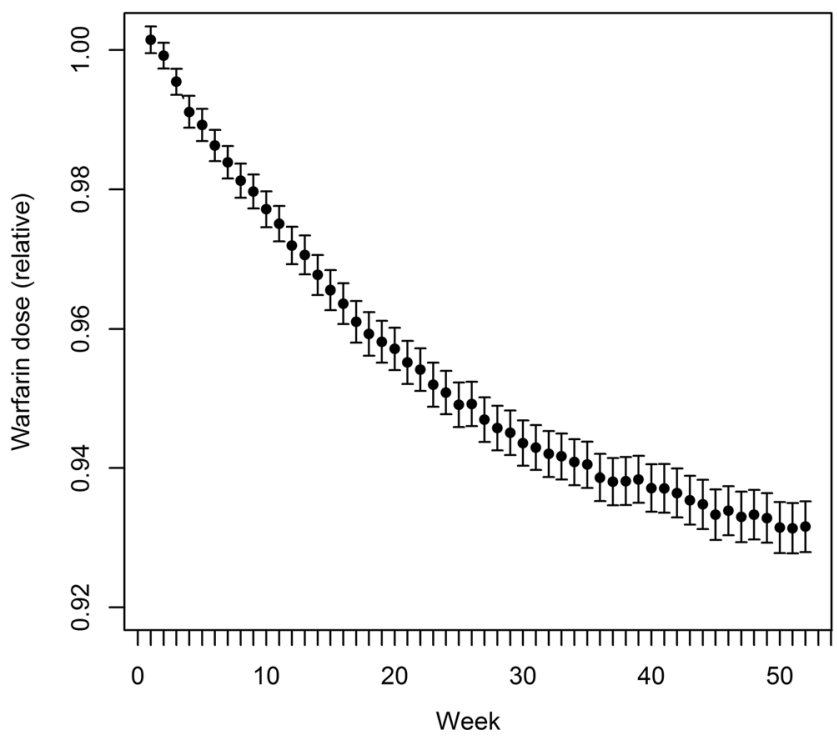

Fig. 4 Changes in dispensed warfarin dose during concomitant simvastatin treatment (means and 95\% confidence intervals)

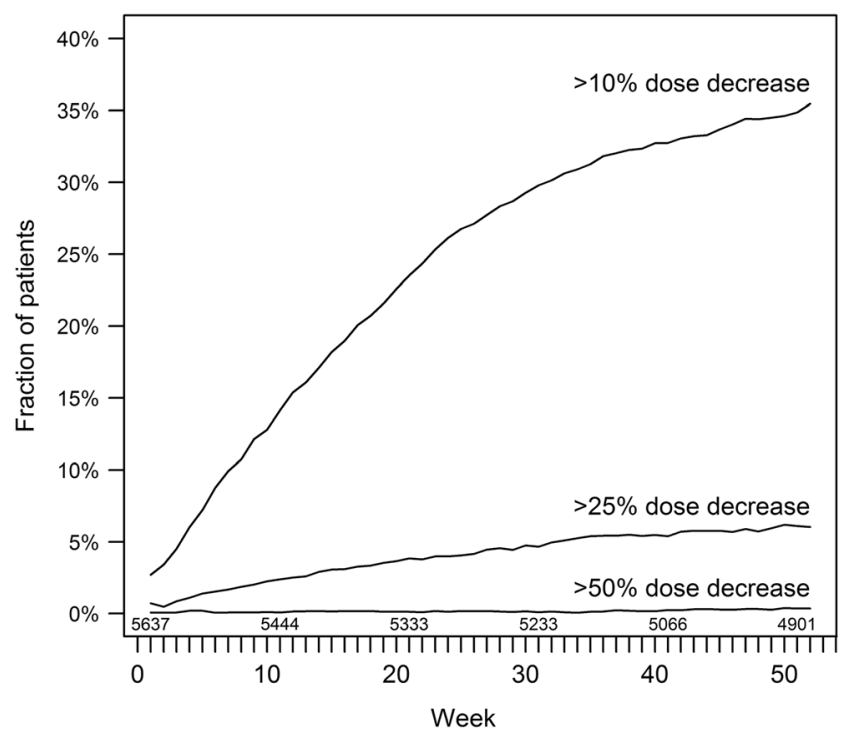

Fig. 5 Fraction of patients with warfarin dose decreases of 10, 25, and $50 \%$ during co-treatment with simvastatin. The numbers at the bottom of the graph indicate the number of patients remaining in study (i.e., still cotreated with warfarin and simvastatin) at different time points

$6.9 \%$ in the second quantile, by $7.0 \%$ in the third quantile, and by $7.9 \%$ in those in the fourth quantile.

\section{Discussion}

In this study, including data from 5637 patients with warfarin, we found that the proportion of patients with an INR above 3 increased from around 8 to $15 \%$ during the first 7 weeks after initiating simvastatin. Warfarin dose requirements were subsequently decreased by $6.8 \%$.

According to the present study, INR was increased by on average $6 \%$. Previous evidence with regard to effects on anticoagulation is limited to two rather small studies by Hickmott et al. and Lin et al. including 29 and 46 patients, respectively. The results indicated an increase in INR by $27 \%$ [9] and 13\% [7]. Lin et al. reported that the number of patients with an INR $>3.0$ increased from 22 to $35 \%$ during simvastatin co-administration. Thus, the effect on anticoagulation was a bit more modest in the present study indicating an increase in INR of $6 \%$. In the present study, initiation of simvastatin decreased warfarin weekly doses by $7 \%$. The results are in line with previous studies indicating a modest decreased requirement of warfarin doses ranging from 3.4 to $9 \%$ [3, 7-10].

In a previous study, we found a significant effect of simvastatin on warfarin doses exclusively in patients with the CYP2C9*3 allele. Unfortunately, we did not have information on the included patients' CYP2C9 genotypes. As patients with the $* 3$ allele are known to have substantially lower warfarin maintenance doses, we instead used the baseline dose of warfarin as a proxy for this variant. However, we could not 
demonstrate a more pronounced interaction effect in individuals with low baseline doses of warfarin, and the trend was indeed opposite to our hypothesis. A weakness of this approach may have been that subjects with the $* 3$ genotype are more prone to have difficulties having a stabile INR and may have changed anticoagulant therapy due to this. In addition, it should be acknowledged that warfarin dose requirements are also influenced by the VKORC1 genotype and a range of other factors unrelated to the CYP2C9 genotype. Consequently, the validity of warfarin dose as a proxy for CYP2C9 enzymatic activity could be questioned.

One possible pharmacokinetic mechanism other than a selective inhibition of the CYP2C*3 variant includes interaction due to competition of CYP3A4-mediated metabolism of Rwarfarin [10]. Pharmacodynamic effects have also been suggested to cause the interaction. Several, but not all, studies have shown that the risk of thrombosis is lower in statin users than in non-users. Hence, simvastatin may have an antithrombotic effect on its own. Simvastatin has been shown to decrease the levels of fibrinogen, factor VII, and plasminogen factor [16] and to decrease platelet aggregation [17].

Using three nationwide registries, we have developed a methodology that previously have proven useful to study drugs that decrease [5] and increase [4] the effect of warfarin anticoagulation. The approach has several strengths especially the high number of subjects and the possibility to study the longitudinal effect of the interaction. One limitation of the present study is that we lack information about the patients' compliance to simvastatin treatment. However, the rather strict inclusion criteria in the present study requiring three additional simvastatin dispensings after the index date may have decreased the influence of non-compliance. Another limitation is that patients with a pronounced interaction effect may have been switched to other anticoagulation and consequently excluded from the analysis. Finally, we did not have access to data on adverse events in the cohort and could not analyze the clinical impact of the interaction effect. However, substantial evidence shows that an INR of 3.0 increases the risk of cerebral hemorrhage and other severe bleeding [18, 19].

In Fig. 5, we present the proportion of patients with different levels of decreases in warfarin doses. Importantly, the changes are calculated separately for each week and not cumulatively. Nevertheless, these changes may theoretically still increase with time due to changes in different time-dependent covariates. To picture this potential statistical bias, we also analyzed the proportions of individuals with different levels of increases in warfarin doses after 1 year after the index date. The proportion of patients with warfarin doses increased by $>$ $11 \%$ was only a sixth $(7 \%)$ of that with doses decreased by $>$ $10 \%(35 \%)$ indicating that such statistical effect was small.

Simvastatin is one of the most extensively used drugs and is often used in individuals also dispensed anticoagulants. The results of the present study therefore have important implications. Although the average effect on warfarin requirements and anticoagulation was modest, the proportion of patients exposed at supratherapeutic INR levels was almost doubled which may have serious consequences with regard to the risk of bleeding. Prescribers should include this information when facing a patient initiating treatment with simvastatin.

In conclusion, initiation of simvastatin resulted in moderately increased INR values and subsequent dose decreases in patients already on warfarin. In order to avoid the increased risk of bleeding, the initiation of simvastatin may be accompanied by closer INR monitoring.

Authors' contribution MLA, JDL, and BM designed the study, interpreted the data, wrote the paper, and approved the final version and have agreed to be accountable for all aspects of the work in ensuring that questions related to the accuracy or integrity of any part of the work have been appropriately investigated and resolved. JDL performed the statistical analysis.

Funding information The study was supported by Bengt Ihres fond, Karolinska Institutet.

Open Access This article is distributed under the terms of the Creative Commons Attribution 4.0 International License (http:// creativecommons.org/licenses/by/4.0/), which permits unrestricted use, distribution, and reproduction in any medium, provided you give appropriate credit to the original author(s) and the source, provide a link to the Creative Commons license, and indicate if changes were made.

\section{References}

1. Fitzmaurice DA, Blann AD, Lip GY (2002) Bleeding risks of antithrombotic therapy. Bmj. 325(7368):828-831

2. Wadelius M, Chen LY, Lindh JD, Eriksson N, Ghori MJ, Bumpstead S et al (2009) The largest prospective warfarin-treated cohort supports genetic forecasting. Blood. 113(4):784-792. https://doi.org/10.1182/blood-2008-04-149070

3. Andersson ML, Lindh JD, Mannheimer B (2013) The impact of interacting drugs on dispensed doses of warfarin in the Swedish population: a novel use of population based drug registers. J Clin Pharmacol 53(12):1322-1327. https://doi.org/10.1002/jcph.174

4. Holm J, Lindh JD, Andersson ML, Mannheimer B (2017) The effect of amiodarone on warfarin anticoagulation: a register-based nationwide cohort study involving the Swedish population. J Thromb Haemost 15(3):446-453. https://doi.org/10.1111/jth.13614

5. Mannheimer B, Andersson ML, Jarnbert-Pettersson H, Lindh JD (2016) The effect of carbamazepine on warfarin anticoagulation: a register-based nationwide cohort study involving the Swedish population. J Thromb Haemost 14(4):765-771. https://doi.org/10.1111/ jth. 13268

6. Holm J, Eiermann B, Eliasson E, Mannheimer B (2014) A limited number of prescribed drugs account for the great majority of drugdrug interactions. Eur J Clin Pharmacol 70(11):1375-1383. https:// doi.org/10.1007/s00228-014-1745-3

7. Lin JC, Ito MK, Stolley SN, Morreale AP, Marcus DB (1999) The effect of converting from pravastatin to simvastatin on the pharmacodynamics of warfarin. J Clin Pharmacol 39(1):86-90 
8. Gage BF, Eby C, Johnson JA, Deych E, Rieder MJ, Ridker PM, Milligan PE, Grice G, Lenzini P, Rettie AE, Aquilante CL, Grosso L, Marsh S, Langaee T, Farnett LE, Voora D, Veenstra DL, Glynn RJ, Barrett A, McLeod H (2008) Use of pharmacogenetic and clinical factors to predict the therapeutic dose of warfarin. Clin Pharmacol Ther 84(3):326-331. https://doi.org/10.1038/clpt.2008. 10

9. Hickmott H, Wynne H, Kamali F (2003) The effect of simvastatin co-medication on warfarin anticoagulation response and dose requirements. Thromb Haemost 89(5):949-950. https://doi.org/10. 1267/THRO03050949

10. Andersson ML, Eliasson E, Lindh JD (2012) A clinically significant interaction between warfarin and simvastatin is unique to carriers of the CYP2C9*3 allele. Pharmacogenomics. 13(7):757-762. https://doi.org/10.2217/pgs. 12.40

11. Journalia, Available from: http://www.journalia.se/. Access date February 2019

12. Auricula, available from: http://www.ucr.uu.se/auricula/. Access date February 2019

13. Wettermark B, Hammar N, Fored CM, Leimanis A, Otterblad Olausson P, Bergman U et al (2007) The new Swedish Prescribed Drug Register-opportunities for pharmacoepidemiological research and experience from the first six months. Pharmacoepidemiol Drug Saf 16(7):726-735. https://doi.org/10. 1002/pds.1294

14. Rosendaal FR, Cannegieter SC, van der Meer FJ, Briet E (1993) A method to determine the optimal intensity of oral anticoagulant therapy. Thromb Haemost 69(3):236-239
15. R Core team. R: a language and environment for statistical computing 2013. http:/www.R-project.org/. Accessed February 2019

16. Krysiak R, Okopien B (2013) Effect of simvastatin on hemostasis in patients with isolated hypertriglyceridemia. Pharmacology. 92(3-4):187-190. https://doi.org/10.1159/000341909

17. Malyszko J, Malyszko JS, Hryszko T, Mysliwiec M (2003) Influence of simvastatin on aspects of thrombogenesis in CAPD patients. Perit Dial Int 23(3):260-266

18. Nelson WW, Wang L, Baser O, Damaraju CV, Schein JR (2015) Out-of-range INR values and outcomes among new warfarin patients with non-valvular atrial fibrillation. Int J Clin Pharm 37(1): 53-59. https://doi.org/10.1007/s11096-014-0038-3

19. Wan Y, Heneghan C, Perera R, Roberts N, Hollowell J, Glasziou P, Bankhead C, Xu Y (2008) Anticoagulation control and prediction of adverse events in patients with atrial fibrillation: a systematic review. Circ Cardiovasc Qual Outcomes 1(2):84-91. https://doi. org/10.1161/circoutcomes. 108.796185

Publisher's note Springer Nature remains neutral with regard to jurisdictional claims in published maps and institutional affiliations. 